\title{
Establishment of mouse neuron and microglial cell co-cultured models and its action mechanism
}

\author{
Bo Zhang ${ }^{1}$, Yunfeng Yang ${ }^{1}$, Jun Tang ${ }^{1}$, Yihao Taoㄹ, Bing Jiang ${ }^{1}$, Zhi Chen ${ }^{1}$, Hua \\ Feng ${ }^{1}$, Liming Yang ${ }^{1}$ and Gang Zhu ${ }^{1}$ \\ ${ }^{1}$ Department of Neurosurgery, Southwest Hospital,Third Military Medical University, Chongqing, China \\ Correspondence to: Liming Yang, email: yangliming212@163.com \\ Gang Zhu, email: gangzhu6666@sina.com
}

Keywords: neuron, microglial cell, OGD, tOGD

Received: February 12, 2017 Accepted: April 15, $2017 \quad$ Published: May 16, 2017

Copyright: Zhang et al. This is an open-access article distributed under the terms of the Creative Commons Attribution License 3.0 (CC BY 3.0), which permits unrestricted use, distribution, and reproduction in any medium, provided the original author and source are credited.

\section{ABSTRACT}

Objective: The objective of this study is to establish a co-culture model of mouse neurons and microglial cells, and to analyze the mechanism of action of oxygen glucose deprivation (OGD) and transient oxygen glucose deprivation (tOGD) preconditioning cell models.

Results: Mouse primary neurons and BV2 microglial cells were successfully cultured, and the OGD and tOGD models were also established. In the co-culture of mouse primary neurons and microglial cells, the cell number of tOGD mouse neurons and microglial cells was larger than the OGD cell number, observed by a microscope. CCK- 8 assay result showed that at $1 \mathrm{~h}$ after treatment, the $O D$ value in the control group is lower compared to all the other three groups $(P<0.05)$. The treatment group exhibited the highest OD value among the four groups. The results observed at $5 \mathrm{~h}$ were consistent with the results at $1 \mathrm{~h}$. Flow cytometry results showed that at $1 \mathrm{~h}$ after treatment the apoptosis percentages is higher in the control group compared to other three groups $(P<0.05)$.

Materials and Methods: Mouse brain tissues were collected and primary neurons cells were cultured. In the meantime mouse BV2 microglia cells were cultured. Two types of cells were co-cultured, and OGD and tOGD cell models were established. There were four groups in the experiment: control group (OGD), treatment group (tOGD+OGD), placebo group (tOGD+OGD+saline) and minocycline intervention group (tOGD+OGD+minocycline). CCK-8 kit was used to detect cell viability and flow cytometry was used to detect apoptosis.

Conclusions: In this study, mouse primary neurons and microglial cells were cocultured. The OGD and tOGD models were established successfully. tOGD was able to effectively protect neurons and microglial cells from damage, and inhibit the apoptosis caused by oxygen glucose deprivation.

\section{INTRODUCTION}

Ischemic stroke, a common clinical disease also known as cerebral infarction, is most likely caused by various reasons and exhibits blockage of cerebral circulation supply, cerebral ischemia and hypoxia, leading to necrosis, as well as the subsequent neuronal functional damage [1]. Cerebral infarction is one of the main causes of death in clinical practice, with poor prognosis and causing a heavy burden of disease [2]. The current treatment options for cerebral infarction are limited. Ineffectiveness, high cost and significant individual differences in response are present in some of the therapies $[3,4]$. Therefore, it has become a hot research topic to search an effective therapy for ischemic stroke.

Oxygen and glucose deprivation (OGD) is a model to simulate human brain infarction by depriving carbohydrates and oxygen from nutrient supply in the cells [5-8]. At present a number of studies have utilized OGD in vitro models to investigate an effective treatment method for ischemic stroke and have shown good results $[9,10]$. Oxygen and glucose deprivation preconditioning refers to the tolerability of tissue to oxygen and glucose deprivation damage, acquired by 
once or multiple times of transient oxygen and glucose deprivation [11-13].

Microglia are resident macrophages in the brain, and are thought to be the major promoter and participant of the brain inflammatory response [14]. Its activation process includes cell proliferation, differentiation, phagocytosis and secretion of cytokines. Recent studies suggest that microglial function depends on the type of stimulation and the different activation states caused by the intensity and the corresponding different immune functional phenotypes, which can simultaneously exert neuroprotective and neurotoxic effects $[14,15]$. In this study, mouse neurons and microglial cells were co-cultured, and OGD and tOGD models were established to investigate the protective effect and the mechanism of action of oxygen glucose deprivation preconditioning on neurons.

\section{RESULTS}

\section{Co-culture of mouse neurons and BV2 microglial cells}

Mouse primary neurons and BV2 microglial cells were successfully cultured in the study. Cells grew in a good condition and were ready for subsequent experiments, as shown in Figure 1.

\section{Co-culture of two types of cells and OGD/tOGD models}

Mouse neurons and microglial cells were cocultured and went through tOGD and OGD experiment. In the same sight of microscope, the number of tOGD mouse neurons and microglial cells was larger than the number of cells under OGD condition, as shown in Figure 2.

\section{Result of CCK-8 cell viability assay}

The result of CCK-8 assay showed that the difference of OD values between control group and the other three groups was statistically significant, with the OD values of all three groups higher compared to control group $(P<0.05)$. In the meantime, the difference between treatment group and minocycline intervention group, and that between placebo group and minocycline intervention group, were both statistically significant $(P<0.05)$. Among the four experimental groups, treatment group exhibited the highest $\mathrm{OD}$ value, as shown in Figure $3 \mathrm{~A}$. The result observed at $5 \mathrm{~h}$ was similar to that at $1 \mathrm{~h}$, with the OD values of all three groups higher compared to control group $(P<0.05)$. The difference between treatment group and minocycline intervention group, and that between placebo group and minocycline intervention group, were both statistically significant $(P<0.05)$, as shown in Figure 3B.

\section{Result of apoptosis detection}

Flow cytometry was used to detect apoptosis of four experimental groups at $1 \mathrm{~h}$ and $5 \mathrm{~h}$, as shown in Figure 4A. The results showed that at $1 \mathrm{~h}$, the difference of apoptosis percentages between control group and the other three groups were statistically significant $(P<0.05)$, with the apoptosis percentages of all three groups lower compared to control group. In the meantime, the difference between treatment group and minocycline intervention group, and that between placebo group and minocycline intervention group, were both statistically significant $(P<0.05)$. The result observed at $5 \mathrm{~h}$ was similar to that at $1 \mathrm{~h}$, with the apoptosis percentage of all three other groups lower than that of control group $(P<0.05)$. The difference of apoptosis percentages between treatment group and minocycline

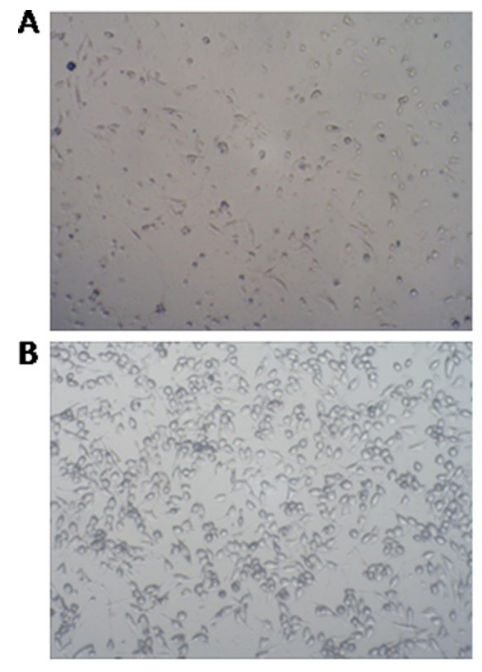

Day 1
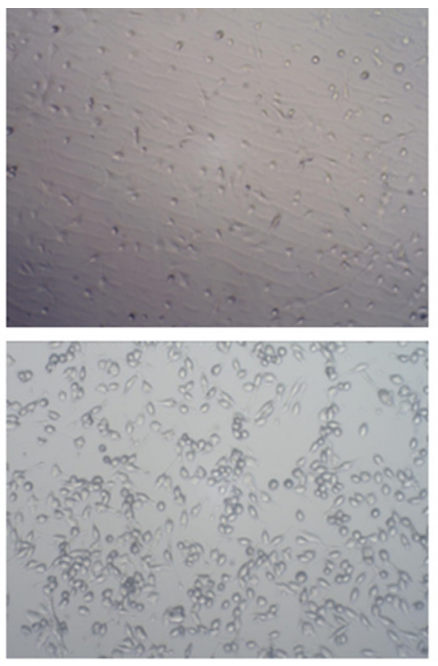

Day 4

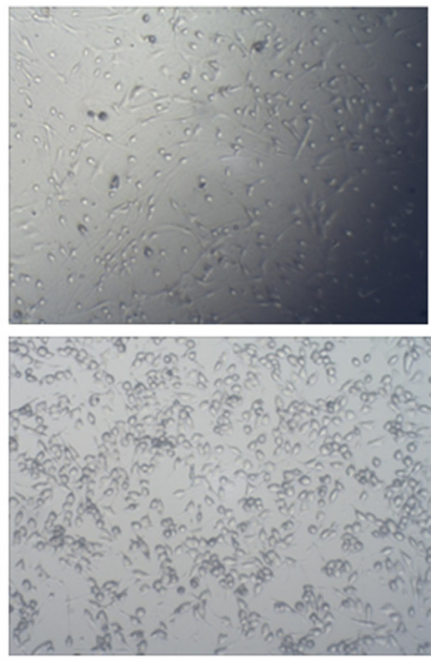

Day 7

Figure 1: Mouse primary neuronal culture and BV2 microglial cell culture. (A) Mouse Primary Neuronal Culture (100×); (B) Mouse BV2 Microglial Cell Culture (100×). 
A

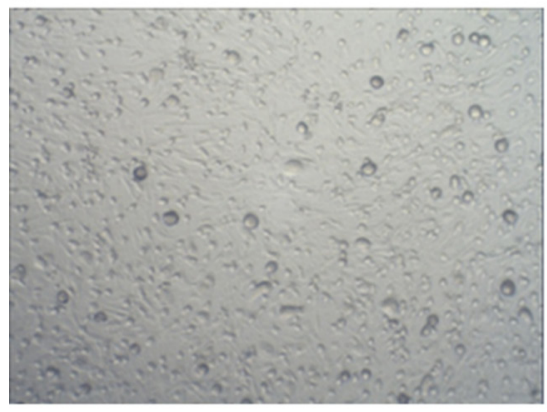

C

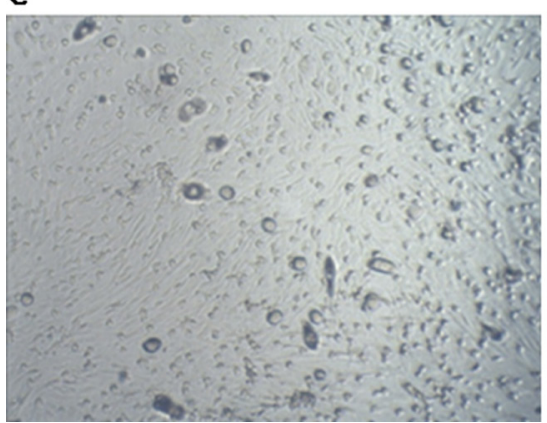

B

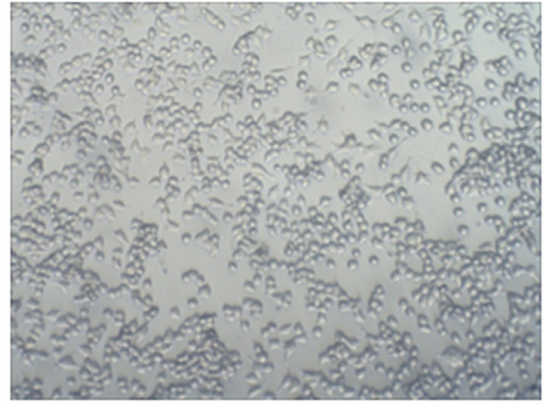

D

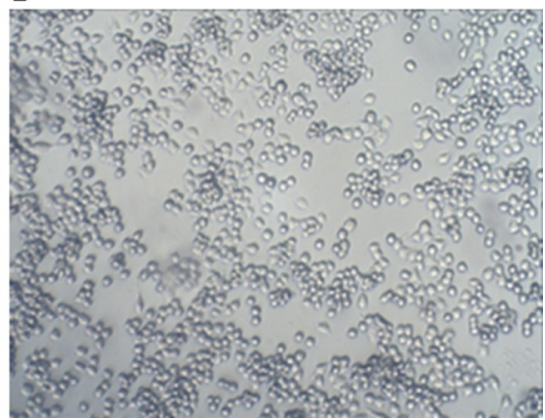

Figure 2: Cell co-culture and tOGD/OGD cell model. (A) Neurons Cells with tOGD $1 \mathrm{~h}(100 \times)$; (B) BV2 Cells tOGD $1 \mathrm{~h}(100 \times)$; (C) Neurons cells with OGD $5 \mathrm{~h}(100 \times)$; (D) BV2 Cells with OGD $5 \mathrm{~h}(100 \times)$.
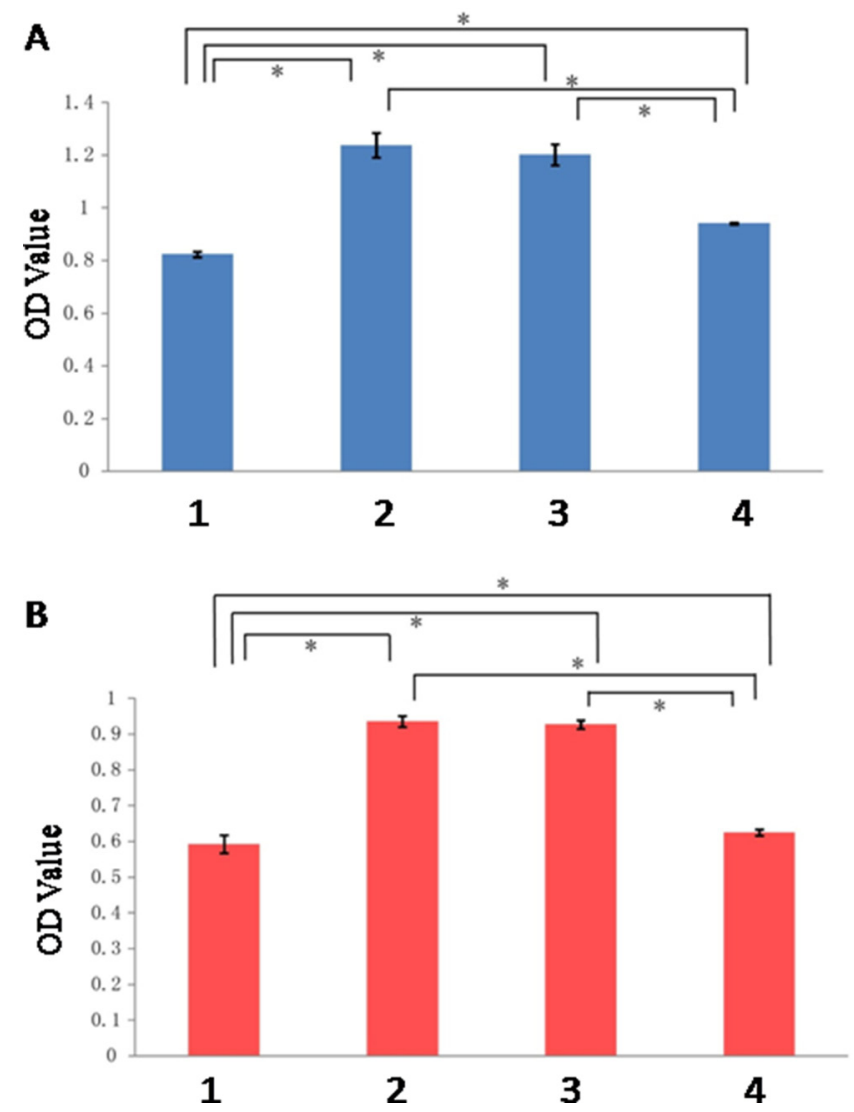

Figure 3: Result of $1 \mathbf{h}$ and 5 h after OGD. (A) 1 h; (B) 5 h. (1: Control Group; 2:Treatment Group; 3: Placebo Group; 4: Minocycline Intervention Group). *The difference between two groups was statistically significant $(P<0.05)$. 
intervention group, and that between placebo group and minocycline intervention group, were both statistically significant $(P<0.05)$, as shown in Figure 4B.

\section{DISCUSSION}

In this study, we successfully co-cultured the mouse primary neurons and microglial cells. We also established the OGD and tOGD models. The results suggested that tOGD was able to effectively protect neurons and microglial cells from damage, and inhibit the apoptosis caused by oxygen glucose deprivation.

The research of OGD models has been reported previously and many mature methods have been established [14-16]. The preparation of OGD models includes two parts: oxygen deprivation and glucose

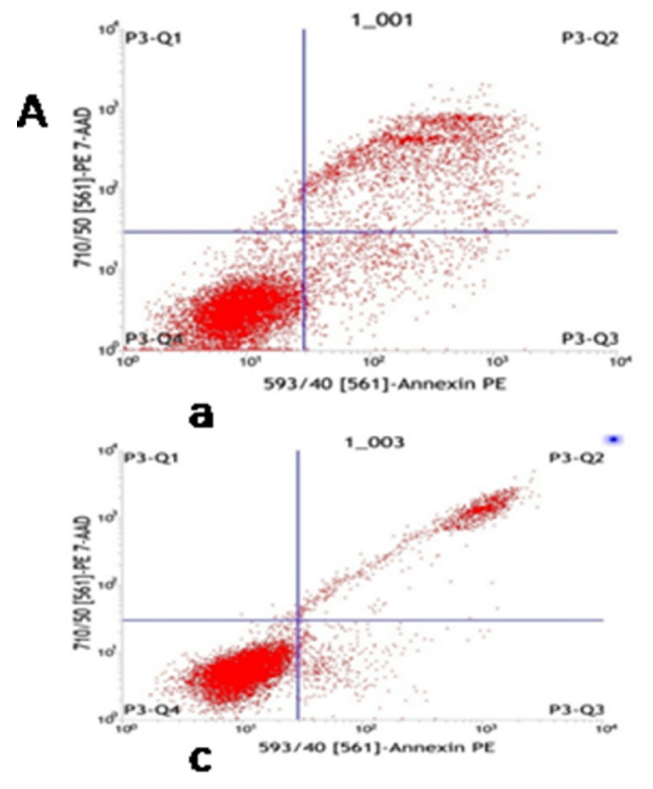

B

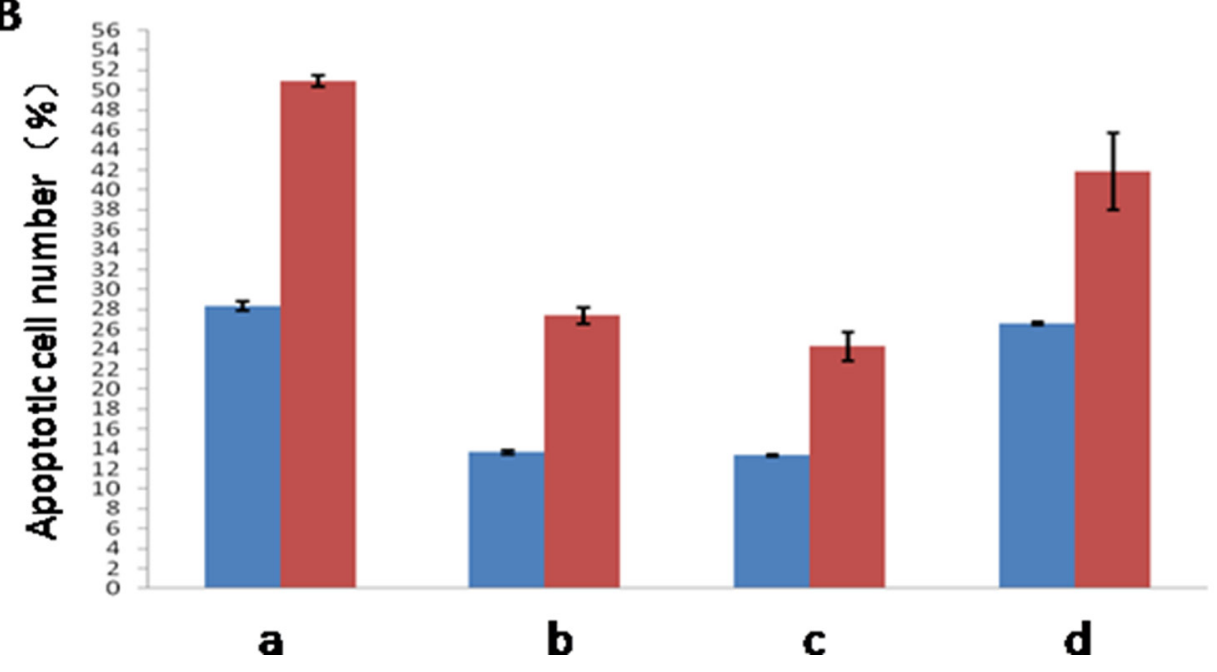

deprivation. Oxygen deprivation is achieved by various methods to lower the oxygen to a minimum amount or undetectable level, while glucose deprivation is conducted by cell culture in glucose free media [17, 18]. In this study, cells were cultured in media without glucose, and oxygen was substituted with nitrogen to achieve an oxygen free condition. This is currently a mature method to prepare OGD models. It can be seen from our result that the OGD and tOGD models were successfully established in the mouse neuron and microglial cell co-culture model.

On the other hand, previous research on the mechanism of action of OGD models less involved apoptosis, while more focused on cell signal transduction and gene expression regulation [19-22]. In our study, we analyzed the neuronal damage resulted from OGD in terms of cellular viability and apoptosis, as well as the effect of

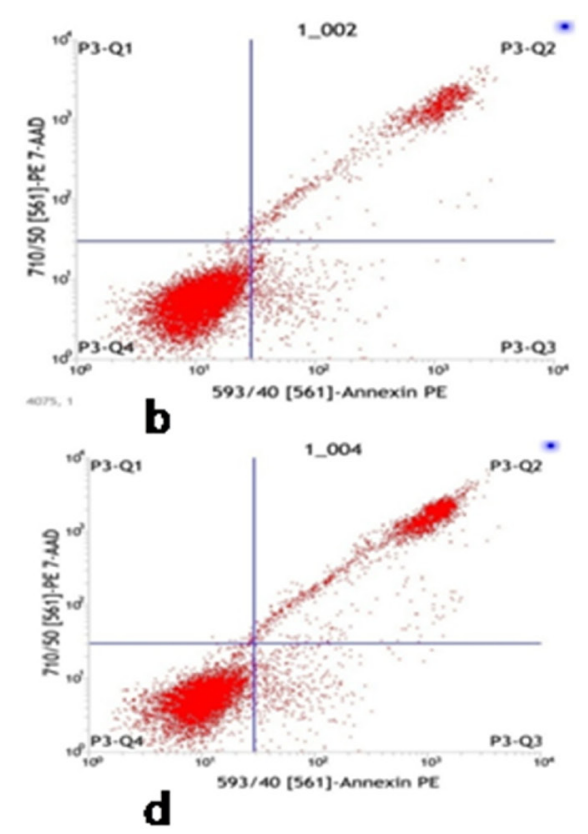

Figure 4: Detection of apoptosis in four experimental groups at different time points. (A) Detection of Apoptosis by Flow Cytometry; (B) Apoptosis in different time point. (a: Control Group; b:Treatment Group; c: Placebo Group; d: Minocycline Intervention Group). 
tOGD on OGD. The results showed that tOGD effectively suppressed the effect of OGD on neurons and microglial cells. Most of neurons and microglial cells were able to grow and proliferate normally following the action of tOGD. In the meantime, preconditioning improved the cell survival rate, shown as cell proliferation rate elevation. tOGD also effectively reduced apoptosis caused by OGD. Therefore, from this perspective, we believed that tOGD might play an important role by inhibiting apoptosis led by OGD.

Two limitations existed in the present study: on the one hand, the mechanism of the effect of tOGD on the cell apoptosis was not be analyzed in depth. One the other hand, we did not performed experiment in vivo.

In conclusion, we found tOGD was able to effectively protect neurons and microglial cells from damage, and inhibit the apoptosis caused by oxygen glucose deprivation, based on the successful establishment of the OGD and tOGD models.

\section{MATERIALS AND METHODS}

\section{Primary culture of mouse neurons}

Forty SPF neonatal mice were purchased from the Experimental Animal Center of the Third Military Medical University. The healthy ICR neonatal mice were sterilized with $75 \%$ alcohol within $24 \mathrm{~h}$ after birth and decapitated in sterile condition. The scalp and skull were cut. Brain tissue was taken out and placed in a dish containing cold D-Hank's solution with pH7.2 and free of calcium and magnesium. Brain tissue was peeled in sterile condition. Cerebellum, hippocampus and medulla were removed. Cortex was isolated. Meninges and blood vessels were carefully peeled off. Tissue was cut into blocks of about $1 \mathrm{~mm}^{3}$ using iris scissors, digested in $0.125 \%$ trypsin for $20 \mathrm{~min}$ at $37^{\circ} \mathrm{C}$ and shaken two to three times. The supernatant was discarded and complete media was added to terminate digestion. Tissues were rinsed twice and pipetted 20 times slightly using the Pasteur pipette. The cell suspension was allowed to stand for $2 \mathrm{~min}$. Cell suspension was collected in a new centrifuge tube and centrifuged at $1000 \mathrm{r} / \mathrm{m}$ at $4^{\circ} \mathrm{C}$ for $10 \mathrm{~min}$. The supernatant was discarded. Cells were resuspended in complete media and filtered with a 200 mesh stainless steel filter. The filtrate was stained using trypan blue and cells were counted using a hemocytometer under a microscope. Cells were inoculated at an intensity of $8.0 \times 10^{4}$ to $1.0 \times 10^{5}$ $\mathrm{ml}$ with $400 \mu \mathrm{l} /$ well into a 24 -well plate or $100 \mu \mathrm{l} /$ well into a 96 -well plate pre-coated with poly-L-lysine. Cells were cultured for $4-6 \mathrm{~h}$ in an incubator at $37^{\circ} \mathrm{C}$ with $5 \%$ $\mathrm{CO}^{2}$. Media was changed with serum-free media. At day 3 of in vitro culture, Ara-C working solution was added to inhibit over-proliferation of non-neuronal cells, and aspirated after $24 \mathrm{~h}$ treatment. Thereafter media was changed by half every three days. Cells collected from day 7-21 of in vitro culture were used for the experiment.

\section{Cell culture of mouse microglial cells}

Mouse BV2 microglial cell line was purchased from the cell bank of Chinese Academy of Science, Shanghai. Cryovials were taken out of the $-80^{\circ} \mathrm{C}$ freezer or liquid nitrogen tank, quickly placed into $37^{\circ} \mathrm{C}$ water bath, and shaken mildly to facilitate the contents thawing. Cell suspension was aspirated from cryovials and added into a centrifuge tube with $5 \mathrm{ml}$ cell culture media containing $10 \%$ fetal bovine serum (FBS). Cells were centrifuged at 800 to $1000 \mathrm{rpm}$ for $5 \mathrm{~min}$ and the supernatant was discarded. Cells was added with $1 \mathrm{ml}$ culture media and pipetted to make suspension. Cells were diluted in cell media containing $20 \% \mathrm{FBS}$, inoculated into a cell culture flask, placed in an incubator at $37^{\circ} \mathrm{C}$ with saturated humidity and 5\% $\mathrm{CO}_{2}$. Culture media was changed after the first $24 \mathrm{~h}$ and the subsequent change was determined based on cell growth condition. Adherent cells were observed under a microscope. With $70 \%$ to $80 \%$ confluence, old media was removed from flask and cells were rinsed with PBS for two to three times. 1Trypson was added to digest cells following a ratio of 1-3 $\mathrm{ml}$ tryspin for a $50 \mathrm{ml}$ flask. When cells became shrank, round and detached in the microscope, the flask was shaken slightly to detach all cells. 2-3 ml complete media was added and pipetted. Cells were collected in a centrifuge tube. The supernatant was removed and culture media was added. Cells were pipetted slightly into cell suspension and inoculated into a sterile culture flask with media following $1: 2$ or $1: 3$ ratio. Cell culture was continued or used for further experiments.

\section{Co-culture of two types of cells model establishment of OGD and tOGD}

BV2 cells were cultured in DMEM high glucose media containing $10 \% \mathrm{FBS}$, at $37^{\circ} \mathrm{C}$ in an incubator with $\mathrm{CO}_{2}$ gas. Cells were sub-cultured every 2-3 days. Cells from passage 3 to 6 were collected for subsequent experiments. Neurons were seeded in different dishes and cells from day 7 to 21 of in vitro culture were used for experiment. Co-culture and transwell culture were conducted depending on the purpose of the experiments. Neurons and BV2 were co-cultured following a 1:2 ratio. 96-well plates were seeded with neurons $3 \times 10^{4} /$ well and BV2 cells $1.5 \times 104 /$ well, placed in an incubator at $37^{\circ} \mathrm{C}$ with saturated humidity and $5 \% \mathrm{CO}_{2}$.

OGD and tOGD models: after cell seeding, the incubator was filled with nitrogen to substitute air and the subsequent experiments were not conducted until a stable condition of $95 \%$ nitrogen and $5 \% \mathrm{CO}_{2}$. Cells were placed in the above condition. The original culture media was changed into DEME media without glucose (Gibco). Cells growing in the original culture condition were used as positive control. Cells were cultured in tOGD condition for $1 \mathrm{~h}$, and in OGD condition for $5 \mathrm{~h}$. Normal culture 
media was used for $24 \mathrm{~h}$ in the normal condition between the two cultures.

\section{Detection of cell viability by CCK-8}

Cell counting kit (CCK-8 kit) was used to detect cell viability and cells were cultured in the same condition as above. There were four groups in the experiment: control group (OGD), treatment group (tOGD+OGD), placebo group (tOGD+OGD+saline) and minocycline intervention group (tOGD+OGD+minocycline). Cells from different treatment groups were counted, adjusted the concentration to $1 \times 10^{5} \mathrm{ml}$, and seeded in a 96-well plate with $100 \mathrm{ul} /$ well. Cells were seeded in triplicate for each treatment group. The 96-well plate was placed in the incubator $\left(37^{\circ} \mathrm{C}\right.$ and $\left.5 \% \mathrm{CO} 2\right)$ and cells were cultured till the appropriate time. $10 \mu \mathrm{CCK}-8$ solution was added in each well and the cell culture plate was incubated for 1-4 h. The absorbance at $450 \mathrm{~nm}$ was detected using a plate reader. Blank wells (culture media and CCK) and control wells (untreated cells, culture media and CCK) were also detected.

\section{Detection of apoptosis}

Cell culture and experiment groups were set up as above. Cells were cultured in a 6 -well plate till $60 \%$ to $70 \%$ confluence. Based on the needs of experiment, cell culture was continued. Cells were aspirated and placed in a centrifuge tube, centrifuged at $2000 \mathrm{rpm}$ for $5 \mathrm{~min}$, rinsed with PBS twice and centrifuged again at $2000 \mathrm{rpm}$ for 5 min. $1 \times 10^{5}$ cells were collected. $5 \mu 17$-AAC staining solution was added in the $50 \mu \mathrm{l}$ binding buffer and mixed. The above 7-ADD staining solution was added in the collected cells and placed in the dark at room temperature for 5-15 min reaction. $450 \mu \mathrm{l}$ binding buffer was later added and mixed. $1 \mu \mathrm{l}$ Annexin V-PE was added, mixed and placed in the dark at room temperature for 5-15 min. Fluorescent microscope observation or flow cytometry was conducted within $1 \mathrm{~h}$. For flow cytometry, FL2 channel was used to detect the orange-red fluorescence of Annexin $\mathrm{V}-\mathrm{PE}$, with excitation wavelength of $488 \mathrm{~nm}$ and emission wavelength of $578 \mathrm{~nm}$. FL3 channel was used to detect the red fluorescence of 7-AAD, with excitation wavelength of $546 \mathrm{~nm}$ and emission wavelength of $647 \mathrm{~nm}$. Normal cells without apoptotic induction were used as control for fluorescence compensation to remove spectral overlap and determine the position of cross-over.

\section{Statistical analysis}

SPSS17.0 software was used to conduct the statistical analysis. Qualitative variables were detected using chi-square test, while quantitative variables were detected by analysis of variance. Difference with $P<0.05$ was considered statistically significant.

\section{ACKNOWLEDGMENTS AND FUNDING}

This work supported by the grant from National Natural Science Foundation of China (NSFC, NO.81571116, and NSFC, NO.81371440) and the National key basic research development (973 Program) of China (2014CB541606).

\section{CONFLICTS OF INTEREST}

We state that we have no conflicts to disclose.

\section{REFERENCES}

1. Leung WY, Jensen MB. Histological quantification of angiogenesis after focal cerebral infarction: a systematic review. ISRN Neurol. 2013:853737.

2. Chang YP, Li L, Xie YM, Zhuang Y. [Clinical outcomes research on parenterally administered kudiezi in treatment of cerebral infarction]. [Article in Chinese]. Zhongguo Zhong Yao Za Zhi. 2013; 38:3155-3160.

3. Liu T, Duan ZG. [Research progress of intervening cerebral infarction by Chinese herbal injection]. [Article in Chinese]. Zhongguo Zhong Xi Yi Jie He Za Zhi. 2013; 33:1434-1437.

4. Okada K, Shirasaka T, Kano H, Okita Y. Mitral valve repair in active infective endocarditis with cerebral infarction. Asian Cardiovasc Thorac Ann. 2013; 21:215-217.

5. Adebiyi A, McNally EM, Jaggar JH. Vasodilation induced by oxygen/glucose deprivation is attenuated in cerebral arteries of SUR2 null mice. Am J Physiol Heart Circ Physiol. 2011; 301:H1360-1368.

6. Chen WW, Yu H, Fan HB, Zhang CC, Zhang M, Zhang C, Cheng Y, Kong J, Liu CF, Geng D. RIP1 mediates the protection of geldanamycin on neuronal injury induced by oxygen-glucose deprivation combined with zVAD in primary cortical neurons. J Neurochem. 2012; 120:70-77.

7. Dennis SH, Jaafari N, Cimarosti H, Hanley JG, Henley JM, Mellor JR. Oxygen/glucose deprivation induces a reduction in synaptic AMPA receptors on hippocampal CA3 neurons mediated by mGluR1 and adenosine A3 receptors. J Neurosci. 2011; 31:11941-11952.

8. Johnsen D, Murphy SJ. Isoflurane preconditioning protects astrocytes from oxygen and glucose deprivation independent of innate cell sex. J Neurosurg Anesthesiol. 2011; 23:335-340.

9. Kritis A, Pourzitaki C, Klagas I, Chourdakis M, Albani M. Proteases inhibition assessment on PC12 and NGF treated cells after oxygen and glucose deprivation reveals a distinct role for aspartyl proteases. PLoS One. 2011; 6:e25950.

10. Lee HJ, Lyu da H, Koo U, Lee SJ, Hong SS, Kim K, Kim KH, Lee D, Mar W. Inhibitory effect of 2-arylbenzofurans from the Mori Cortex Radicis (Moraceae) on oxygen glucose deprivation (OGD)-induced cell death of SH-SY5Y cells. Arch Pharm Res. 2011; 34:1373-1380. 
11. Liu J, Litt L, Segal MR, Kelly MJ, Yoshihara HA, James TL. Outcome-related metabolomic patterns from 1H/31P NMR after mild hypothermia treatments of oxygenglucose deprivation in a neonatal brain slice model of asphyxia. J Cereb Blood Flow Metab. 2011; 31:547-559.

12. Liu R, Zhang L, Lan X, Li L, Zhang TT, Sun JH, Du GH. Protection by borneol on cortical neurons against oxygen-glucose deprivation/reperfusion: involvement of anti-oxidation and anti-inflammation through nuclear transcription factor kappaappaB signaling pathway. Neuroscience. 2011; 176:408-419.

13. Martin-de-Saavedra MD, del Barrio L, Canas N, Egea J, Lorrio S, Montell E, Verges J, Garcia AG, Lopez MG. Chondroitin sulfate reduces cell death of rat hippocampal slices subjected to oxygen and glucose deprivation by inhibiting p38, NFkappaB and iNOS. Neurochem Int. 2011; 58:676-683.

14. Graeber MB. Changing face of microglia. Science. 2010; 330:783-788.

15. Vinet J, Weering HR, Heinrich A, Kälin RE, Wegner A, Brouwer N, Heppner FL, Rooijen Nv, Boddeke HW, Biber K. Neuroprotective function for ramified microglia in hippocampal excitotoxicity. J Neuroinflammation. 2012; 9:27.

16. Neuhaus W, Burek M, Djuzenova CS, Thal SC, Koepsell H, Roewer N, Forster CY. Addition of NMDA-receptor antagonist MK801 during oxygen/glucose deprivation moderately attenuates the upregulation of glucose uptake after subsequent reoxygenation in brain endothelial cells. Neurosci Lett. 2012; 506:44-49.

17. Peng S, Kalikiri P, Mychaskiw G 2nd, Zhang D, Zhang Y, Liu GJ, Wang GL, Shen ZY. Sevoflurane postconditioning ameliorates oxygen-glucose deprivation-reperfusion injury in the rat hippocampus. CNS Neurosci Ther. 2011; 17:605-611.
18. Qi H, Han Y, Rong J. Potential roles of PI3K/Akt and Nrf2Keap1 pathways in regulating hormesis of Z-ligustilide in PC12 cells against oxygen and glucose deprivation. Neuropharmacology. 2012; 62:1659-1670.

19. Qian Y, Guan T, Tang X, Huang L, Huang M, Li Y, Sun H. Maslinic acid, a natural triterpenoid compound from Olea europaea, protects cortical neurons against oxygenglucose deprivation-induced injury. Eur J Pharmacol. 2011; 670:148-153.

20. Radley E, Akram A, Grubb BD, Gibson CL. Investigation of the mechanisms of progesterone protection following oxygen-glucose deprivation in organotypic hippocampal slice cultures. Neurosci Lett. 2012; 506:131-135.

21. Ricci L, Valoti M, Sgaragli G, Frosini M. Taurine-like GABA aminotransferase inhibitors prevent rabbit brain slices against oxygen-glucose deprivation-induced damage. Amino Acids. 2012; 42:2139-2147.

22. Saini MG, Bix GJ. Oxygen-glucose deprivation (OGD) and interleukin-1 (IL-1) differentially modulate cathepsin B/L mediated generation of neuroprotective perlecan LG3 by neurons. Brain Res. 2012; 1438:65-74.

23. Sharma J, Nelluru G, Wilson MA, Johnston MV, Hossain MA. Sex-specific activation of cell death signalling pathways in cerebellar granule neurons exposed to oxygen glucose deprivation followed by reoxygenation. ASN Neuro. 2011; 3.

24. Talbot JD, David G, Barrett EF, Barrett JN. Calcium dependence of damage to mouse motor nerve terminals following oxygen/glucose deprivation. Exp Neurol. 2012; 234:95-104. 